\title{
Histamine-releasing factor enhances food allergy
}

\author{
Tomoaki Ando, ${ }^{1,2}$ Jun-ichi Kashiwakura, ${ }^{1}$ Naoka Itoh-Nagato, ${ }^{3}$ Hirotaka Yamashita, ${ }^{4}$ Minato Baba, ${ }^{5}$ Yu Kawakami, ${ }^{5}$ \\ Shih Han Tsai, ${ }^{6,7}$ Naoki Inagaki, ${ }^{4}$ Kiyoshi Takeda, ${ }^{6,7}$ Tsutomu Iwata, ${ }^{8}$ Naoki Shimojo, ${ }^{9}$ Takao Fujisawa, ${ }^{10}$ Mizuho Nagao, ${ }^{11}$ \\ Kenji Matsumoto, ${ }^{12}$ Yuko Kawakami, ${ }^{5}$ and Toshiaki Kawakami ${ }^{5,13}$
}

\begin{abstract}
'Laboratory for Cytokine Regulation, RIKEN Center for Integrative Medical Sciences (RIKEN IMS), Yokohama, Japan. ${ }^{2}$ Atopy Research Center, Graduate School of Medicine, Juntendo University, Tokyo, Japan. ${ }^{3}$ Laboratory for Intestinal Ecosystem, RIKEN IMS, Yokohama, Japan. " ${ }^{4}$ aboratory of Pharmacology, Department of Bioactive Molecules, Gifu Pharmaceutical University, Gifu, Japan. ${ }^{5}$ Division of Cell Biology, La Jolla Institute for Allergy and Immunology, La Jolla, California, USA. ${ }^{6}$ Laboratory of Immune Regulation, Department of Microbiology and Immunology, Graduate School of Medicine, WPI Immunology Frontier Research Center, Osaka University, Suita, Osaka, Japan. ${ }^{7}$ Core Research for Evolutional Science and Technology, Japan Science and Technology Agency, Saitama, Japan. ${ }^{8}$ Department of Education for Childcare, Faculty of Child Studies, Tokyo Kasei University, Tokyo, Japan. ${ }^{9}$ Department of Pediatrics, Graduate School of Medicine, Chiba University, Chiba, Japan. ${ }^{10} \mathrm{Department}$ of Pediatrics and Allergy and "IInstitute for Clinical Research, Mie National Hospital, Tsu, Mie, Japan. ${ }^{2}$ Department of Allergy and Clinical Immunology, National Research Institute for Child Health and Development, Tokyo, Japan.
\end{abstract} ${ }^{13}$ Department of Dermatology, UCSD, School of Medicine, La Jolla, California, USA.

Food allergy occurs due to IgE- and mast cell-dependent intestinal inflammation. Previously, we showed that histaminereleasing factor (HRF), a multifunctional protein secreted during allergy, interacts with a subset of IgE molecules and that the HRF dimer activates mast cells in an HRF-reactive IgE-dependent manner. In this study, we investigated whether HRF plays any role in food allergy. Specifically, we determined that prophylactic and therapeutic administration of HRF inhibitors that block HRF-IgE interactions reduces the incidence of diarrhea and mastocytosis in a murine model of food allergy. Food allergy-associated intestinal inflammation was accompanied by increased secretion of the HRF dimer into the intestine in response to proinflammatory, Th2, and epithelial-derived cytokines and HRF-reactive IgE levels at the elicitation phase. Consistent with these data, patients with egg allergy had higher blood levels of HRF-reactive IgE compared with individuals that were not hypersensitive. Successful oral immunotherapy in egg-allergy patients and food-allergic mice reduced HRFreactive IgE levels, thereby suggesting a pathological role for HRF in food allergy. Together, these results suggest that antigen and HRF dimer amplify intestinal inflammation by synergistically activating mast cells and indicate that HRF has potential as a therapeutic target in food allergy.

\section{Introduction}

The prevalence of food allergy has been dramatically increasing for the last few decades $(1,2)$. Six to eight percent of children under the age of 3 have food allergies, and nearly 4 percent of adults have them. Symptoms of food allergy range from itching, hives, and diarrhea to life-threatening anaphylaxis. Currently, there is no cure for this disease, although allergen-specific immunotherapy can successfully treat some patients (3-6). Basic studies have provided insights into the pathophysiology using various animal models $(7,8)$. Brandt et al. showed that exposure to repeated doses of intragastric (i.g.) OVA induced acute diarrhea in OVA/alum-sensitized mice, which is associated with increased small intestinal permeability, eosinophilia, and mastocytosis; this type 2 inflammation depends largely on mast cells, IgE and highaffinity IgE receptor (Fc\&RI), serotonin, platelet-activating factor, IL-4, and IL-9 $(9,10)$. Thus, the "classical pathway" of anaphylaxis (11) seems essential in this model, where exposure to allergen allows

\section{Related Commentary: p. 4238}

Authorship note: T. Ando and J. Kashiwakura contributed equally to this study. Conflict of interest: The authors have declared that no conflict of interest exists. Submitted: July 27, 2017; Accepted: September 19, 2017.

Reference information: / Clin Invest. 2017;127(12):4541-4553.

https://doi.org/10.1172/JCI96525. allergen-specific IgE to crosslink FceRI on the surface of mast cells and basophils to activate the cells, ultimately leading to the release of various anaphylactogenic mediators. The clinical importance of IgE in human food allergy is supported by the efficacy of omalizum$\mathrm{ab}$, a humanized anti-IgE monoclonal Ab that blocks IgE binding to FceRI (12), in facilitating oral desensitization (13).

Histamine-releasing factor (HRF), also known as translationally controlled tumor protein (TCTP) and fortilin, is a highly conserved protein with both intracellular and extracellular functions $(14,15)$. As an extracellular protein, HRF can stimulate histamine release and IL-4 and IL-13 production from IgEsensitized basophils and mast cells (16). HRF-like activities were found in bodily fluids during allergic reactions, thus implicating HRF in allergic diseases $(17,18)$. However, since HRF has essential intracellular functions, including cell cycle progression, proliferation, survival, and malignant transformation of a variety of cell types (14), it has been a challenge to elucidate its extracellular roles separately from intracellular functions. We recently found that approximately $25 \%$ of $\operatorname{IgE}$ molecules can bind to HRF via their Fab interactions with 2 binding sites within the HRF molecule (19). HRF can form a dimer and crosslink IgEs. Using IgEbinding fragments, such as GST-tagged N-terminal 19 amino acids (GST-N19) or monomeric HRF (HRF-2CA), as competitive HRF inhibitors, we successfully dissected the extracellular functions and demonstrated an essential role for HRF in promoting skin 
A

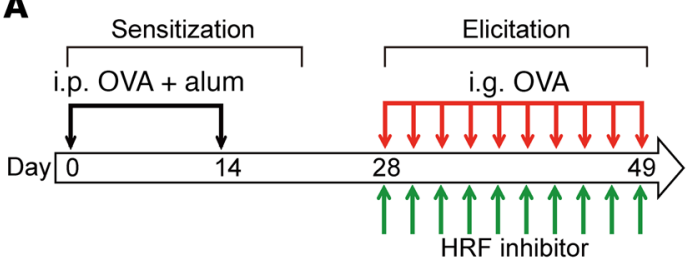

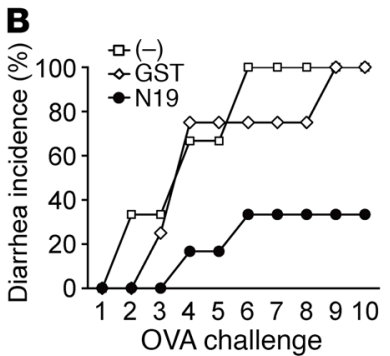

D

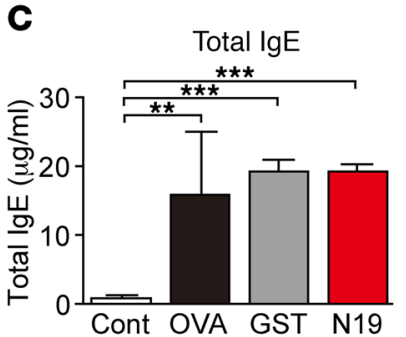

Total IgG1

HRF
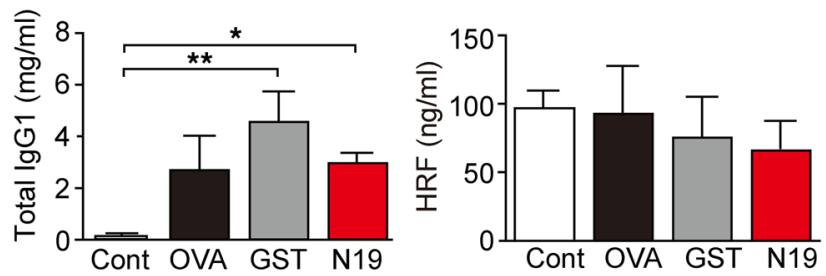

E
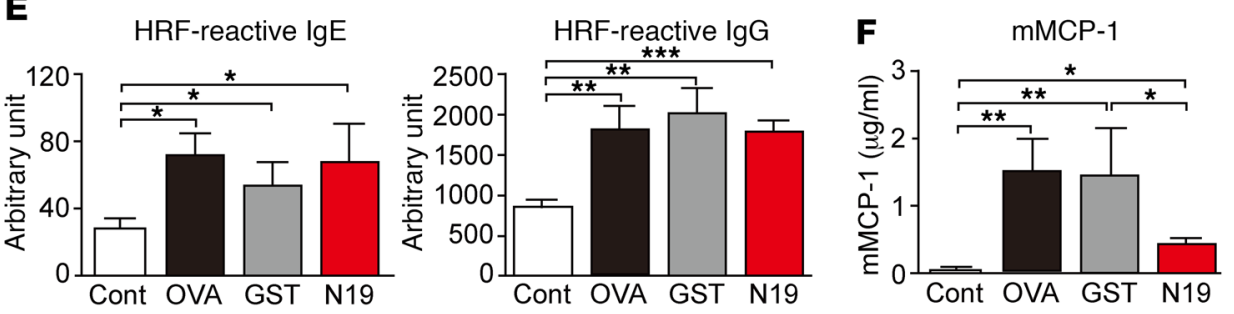

G
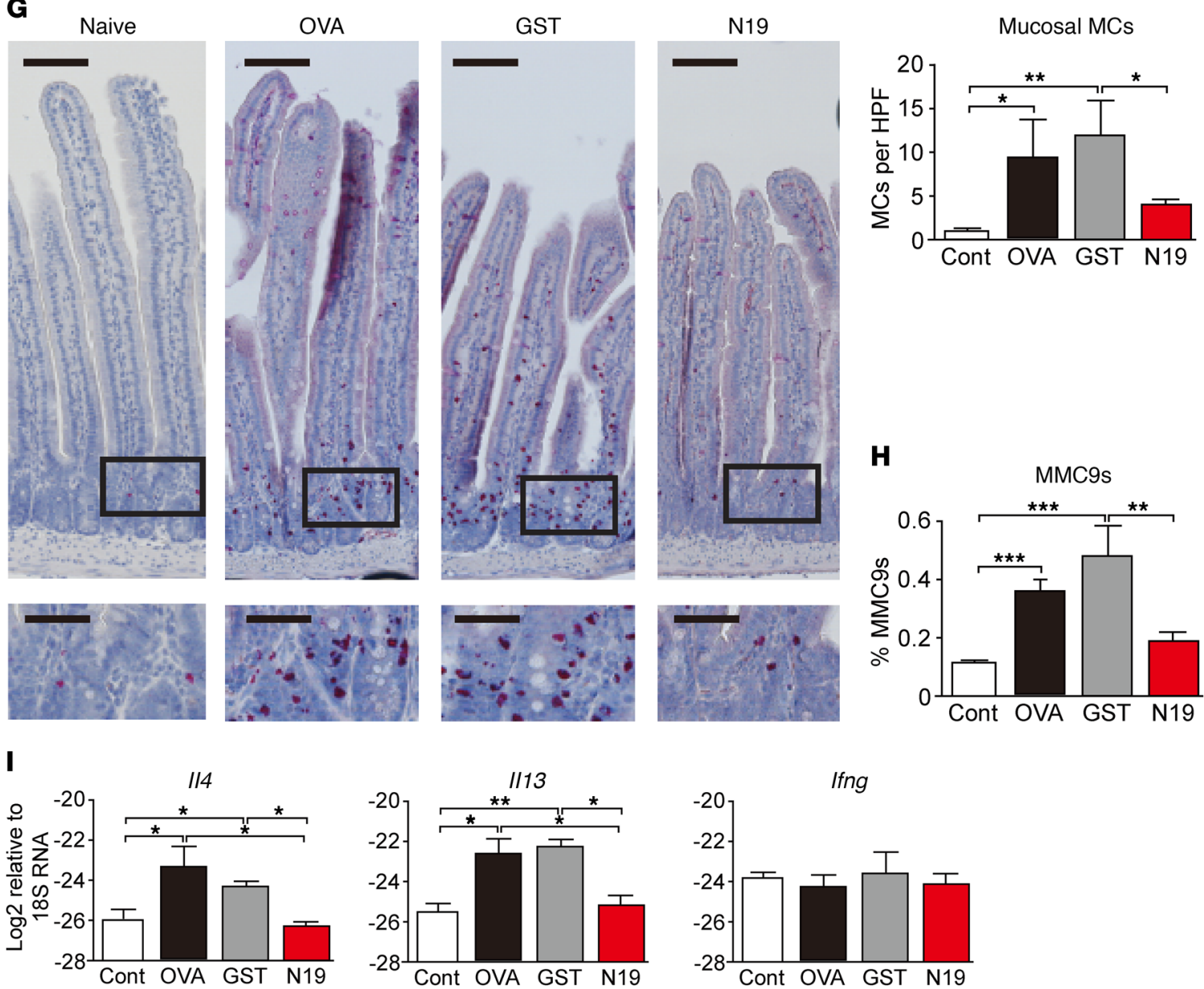
Figure 1. GST-N19 inhibits the development of diarrhea and intestinal mastocytosis in murine food allergy. Mice were i.p. sensitized with OVA ( $50 \mu \mathrm{g} /$ mouse) plus alum on days 0 and 14 . From day 28 , mice were i.g. challenged with OVA $(50 \mathrm{mg}) 3$ times a week. Sensitized mice were either nonpretreated (-) or i.g. pretreated with $100 \mu \mathrm{g}$ of CST or GST-N19 (N19). (A) Procedure schematic. (B) The development of diarrhea was monitored for 90 minutes after each OVA challenge. $n=5$ for nonsensitized group; $n=3$ for inhibitor nonpretreated group; $n=4$ for GST group; $n=6$ for N19 group. Data are representative of at least 3 independent experiments. log-rank test: $P$ $=0.028$ (GST vs. GST-N19), $P=0.036$ (control vs. GST-N19). Nonsensitized mice did not exhibit diarrhea after OVA gavages (not shown). (C-E) Total IgE, IgG1, HRF, and HRF-reactive IgE and IgG in sera were measured by ELISA. (F) Serum mMCP-1 was also measured by ELISA. (G) Sections of jejunum were stained with chloroacetate esterase to identify mast cells. Sections indicated by rectangles are enlarged in lower rows. Scale bars: $100 \mu \mathrm{m}$; $50 \mu \mathrm{m}$ (insets). (H) Flow cytometry was performed to enumerate MMC9s ( inn $^{-}{ }^{-}-\mathrm{Kit}^{+} \mathrm{ST2} 2^{+} \mathrm{IL}-$ 17RB-Integrin $\beta 7^{\circ}$ ) in jejunum. $n=14$ for nonsensitized group; $n=15$ for inhibitor nonpretreated group; $n=9$ for CST group; $n=9$ for CST-N19 group, pooled data of 3 independent experiments. (I) Cytokine mRNAs in jejunum were quantified by qRT-PCR. ${ }^{*} P<0.05 ;{ }^{* *} P<0.01$; ${ }^{* *} P<0.001$, ANOVA with Tukey's multiple comparison. Cont, control.

hypersensitivity and airway inflammation (19). In this study, we investigate whether HRF plays a role in a murine model of food allergy. We also evaluate blood levels of HRF and HRF-reactive Ab titers in egg-allergy patients during a clinical trial of oral immunotherapy (OIT) and murine models of OIT.

\section{Results}

$H R F$ promotes intestinal inflammation during the elicitation phase of murine food allergy. Mice immunized i.p. with OVA in the presence of alum were repeatedly challenged with i.g. gavages of OVA, leading to the development of diarrhea and type 2 inflammation in the small intestine $(9,10)$. Initial diarrheal events occurred mostly from the second to the sixth challenge (Figure 1, A and B). As shown previously (9), serum levels of total IgE and IgG1 as well as OVA-specific IgE and IgG were increased in diarrheal mice, compared with nonsensitized, OVA-challenged control mice (Figure $1 \mathrm{C}$ and data not shown). Interestingly, serum levels of HRFreactive $\operatorname{IgG}$ and $\mathrm{HRF}$-reactive $\mathrm{IgE}$, but not $\mathrm{HRF}$, were also increased (Figure 1, D and E). When sensitized mice were pretreated i.g. with an HRF inhibitor, GST-N19, before each OVA challenge, the onset of diarrhea was delayed and its incidence was reduced (Figure 1, A and B). Consistent with the established role of mast cells in this model (9), the increased serum level of the mast cell protease mMCP-1 was sustained for more than 8 hours after OVA challenges and reduced by GST-N19 pretreatment (Figure $1 \mathrm{~F}$ and data not shown). Furthermore, HRF inhibitor reduced the number of mucosal mast cells found in jejunum (Figure 1G). Flow cytometry confirmed increased IL-9-producing mucosal mast cells (MMC9s), which are precursors to granular mucosal mast cells and critical in this model (20), in food allergic mice, and HRF inhibitor reduced MMC9s in jejunum (Figure 1H). A robust correlation was found between diarrhea occurrence and numbers of mucosal mast cells (Spearman's correlation, $r=0.82, P<0.0001$ ), but numbers of submucosal mast cells were not increased by OVA challenges (Supplemental Figure 1; supplemental material available online with this article; https://doi.org/10.1172/JCI96525DS1 and data not shown). Transcriptome and quantitative reverse- transcriptase PCR (qRT-PCR) analyses of jejunum (Figure 1I and data not shown) showed increased Th2 cytokine mRNAs (Il4 and Il13) in diarrheal mice, which was repressed in inhibitorpretreated mice. In contrast, levels of total IgE, IgG1, IgG2a, or HRF-reactive IgE and IgG were not reduced by HRF inhibitor (Figure 1, $\mathrm{C}$ and $\mathrm{E}$, and data not shown). The i.g. administration of a synthetic N19 peptide or HRF-2CA, which is a monomeric mutant of mouse HRF (19), in place of GST-N19 exhibited similar inhibitory effects on diarrhea occurrence, intestinal inflammation, mucosal mast cells, and serum mMCP-1 levels, but failed to affect levels of IgE, IgG1, or HRF-reactive IgE and IgG (Supplemental Figure 1).

While allergen-induced increases in $\operatorname{IgE}$ and $\operatorname{IgG}$ levels were not changed in HRF inhibitor-treated versus -nontreated mice, the efficacy of HRF inhibitors suggested that they suppress the allergen-triggered elicitation phase of food allergy by targeting mast cells. We next tested whether HRF inhibitors affect the sensitization phase of food allergy. Pretreatment with HRF inhibitor before sensitization with OVA plus alum did not affect diarrhea occurrence or levels of IgE or IgG1 (Supplemental Figure 2), indicating that $\mathrm{HRF}$ regulates the elicitation, but not sensitization, phase in this food allergy model.

$H R F$ inhibitors suppress allergic diarrhea via FcERI. A crucial role for FceRI in food allergy was previously demonstrated in this and other murine models $(9,21)$. We confirmed it using FceRIa ${ }^{-/}$ mice, as allergic diarrhea was severely delayed and suppressed in $\mathrm{FceRIa}^{-/-}$mice. Accordingly, numbers of mucosal mast cells were low, and more importantly, effects of HRF inhibitor were not observed in FceRIa ${ }^{--}$mice (Supplemental Figure 3A).

Since the IgG Fc receptor Fc $\gamma$ RIIB inhibits FceRI-mediated mast cell activation (22) and is critical for the efficacy of allergy immunotherapy (23), we also tested the role of this receptor in food allergy using $\mathrm{FcgRIIB}^{-/-}$mice. Allergic diarrhea was seen in $\mathrm{FcgRIIB}^{-/-}$mice similar to that in WT mice, and HRF inhibitor suppressed allergic diarrhea in the mutant mice (Supplemental Figure 3B). Ectonucleotide pyrophosphatase-phosphodiesterase 3 (E-NPP3, also known as $\mathrm{CD} 203 \mathrm{c}$ ) also negatively regulates FceRI-induced activation (24). Indeed, Enpp3 $3^{--}$mice exhibited enhanced allergic diarrhea accompanied by weight loss as compared with WT control. Again, HRF inhibitor suppressed allergic diarrhea and weight loss in Enpp3 $3^{--}$mice (Supplemental Figure 3C). These results collectively support the notion that HRF promotes allergic diarrhea and type 2 inflammation via FceRI.

Mucosal mast cells from allergic mice are activated by allergen and $H R F$. Reactivity of ex vivo mucosal mast cells in the small intestine was investigated in a new assay termed mast cell activation test (MCAT). When cell mixtures containing mucosal mast cells derived from nonsensitized mice were stimulated with OVA, few mast cells were activated (Figure 2, A and B). In contrast, $2.48 \%$ $\pm 0.136 \%$ (mean $\pm \mathrm{SEM}$ ) of $\mathrm{c}-\mathrm{Kit}^{+}$mucosal mast cells derived from allergic mice expressed an activation marker, lysosomalassociated membrane protein 1 (LAMP-1), on their cell surface without stimulation, and they were further activated by OVA in a dose-dependent manner (Figure 2, A and B). Since mucosal mast cells derived from both nonsensitized and allergic mice were similarly activated by anti-IgE Ab, FceRI molecules in both mice should have been occupied by IgE in vivo. Differential reactivity of the cells to OVA indicated that OVA-specific IgE molecules occu- 
A

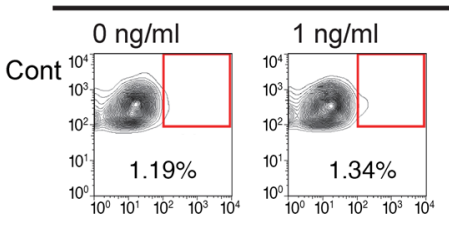

\section{OVA}
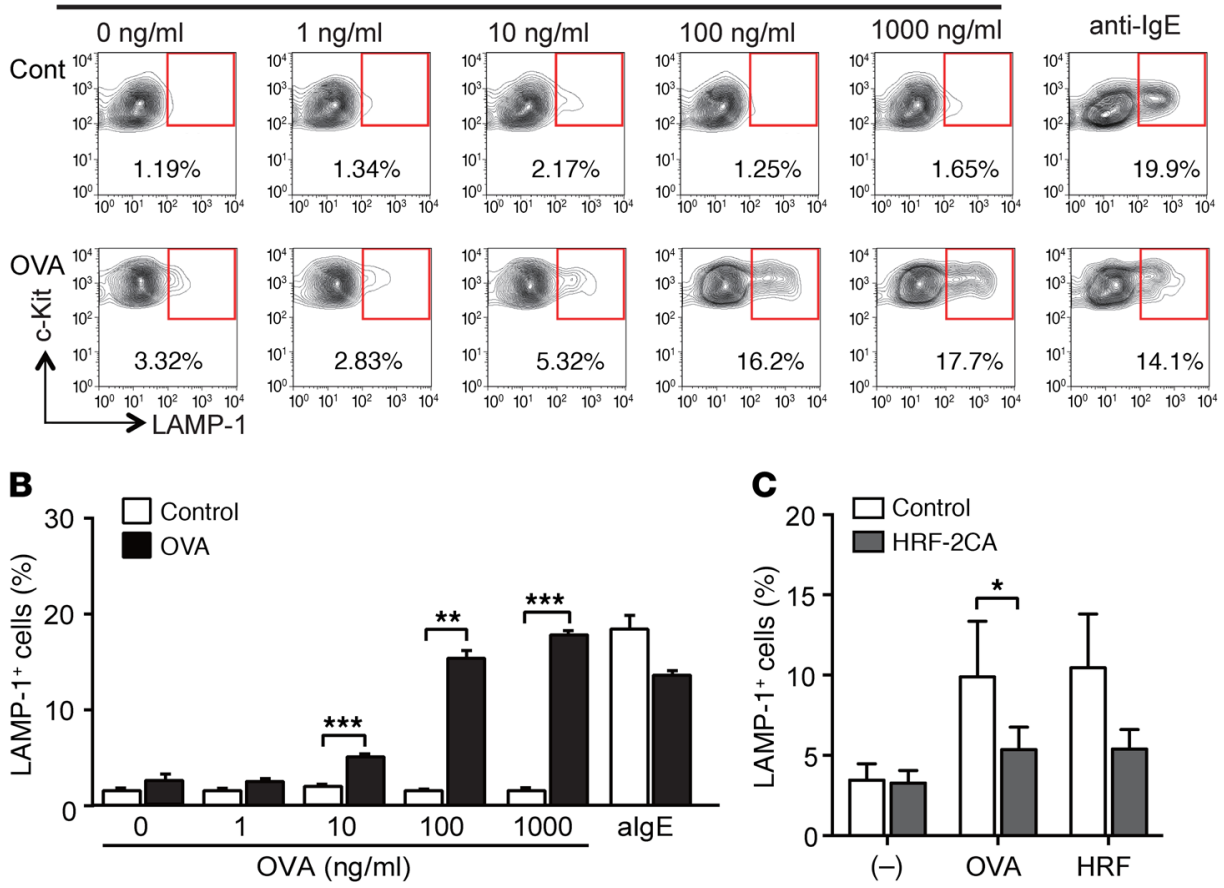

Figure 2. Mucosal mast cells can be activated ex vivo by antigen. Cells were released with EDTA from small intestines of nonsensitized/ OVA-challenged (Cont) or OVA-sensitized/ OVA-challenged (OVA) mice, and mononuclear cells were selected. The cells were incubated for 15 minutes at $37^{\circ} \mathrm{C}$ with OVA or anti-lgE $\mathrm{Ab}$, and activation of $\mathrm{Kit}^{+}$mast cells was measured by flow cytometry (A and $\mathbf{B}$ ). ${ }^{* *} P<0.01$; ${ }^{* *} P<0.001$, Student's $t$ test. $n=2$, representative of 2 independent experiments. (C) Mucosal cells derived from OVA-sensitized/OVA-challenged mice were stimulated with PBS $(n=4)$, OVA $(10 \mathrm{ng} / \mathrm{ml}$, $n=4)$, or HRF dimer $(100 \mu \mathrm{g} / \mathrm{ml}, n=2)$ in the presence or absence of HRF-2CA $(300 \mu \mathrm{g} / \mathrm{ml})$. ${ }^{*} P<0.05$, Student's $t$ test. pied a substantial proportion of FceRI on mast cells in allergic mice, whereas FceRI in nonsensitized mice was occupied by nonspecific IgE. Recombinant dimeric, but not monomeric, HRF activated ex vivo mucosal mast cells (Supplemental Figure 4, A and B). Moreover, HRF inhibitors (i.e., HRF-2CA and N19 peptide) significantly suppressed HRF-induced as well as OVA-induced activation of mucosal mast cells derived from allergic mice (Figure $2 \mathrm{C}$ and data not shown). These ex vivo observations of OVA-induced mast cell activation likely reflect what happened in the small intestine of food-allergic mice.

$H R F$ dimer increases in the small intestine of allergic mice. Disulfide-linked HRF dimers bind to certain IgE molecules, leading to crosslinking of IgE-bound FceRI molecules and their consequent activation (Supplemental Figure 4, A and B, and ref. 19). We thus quantified HRF multimers in inflammatory sites. Jejunal tissues were harvested 24 hours after the tenth OVA challenge, homogenized, and extracted with PBS for a new ELISA to quantify HRF multimers (Supplemental Figure 4C). Three-fold more amounts of HRF multimers were found in allergic than in control mice (Figure 3A), and the total amount of HRF and the ratio of $\mathrm{HRF}$ multimers to total $\mathrm{HRF}$ were also increased in allergic mice (Figure 3, B and C). Consistent with this, Western blot analysis showed that the small intestine of allergic mice had significantly higher ratios of dimeric to monomeric HRF than those of control mice and the ratios were reduced by HRF inhibitor (Figure 3D). The increased multimer-to-monomer ratio was likely due to oxidative stress under allergic conditions $(25,26)$. These results suggest that HRF dimer/multimers promote mast cell activation in the small intestine of allergic mice.

HRF inhibitors target mast cells. We next tested whether HRF inhibitors target mucosal mast cells. To this end, mice with allergic diarrhea were i.g. gavaged with GST-N19 or GST and their location was identified 1 hour after their gavage. Confocal microscopy showed that $71.4 \% \pm 0.64 \%$ of jejunal $\mathrm{IgE}^{+}$cells were also positive for mMCP-1, while other mast cell markers such as mMCP-4 or mMCP- 6 were positive for less than $20 \%$ of $\operatorname{IgE}^{+}$ cells (data not shown). We found that about $65 \%$ of $\operatorname{IgE}^{+} \mathrm{mMCP}^{-}{ }^{+}$ mast cells were colocalized with GST-N19, whereas only $34 \%$ of IgE $\mathrm{mMCP}^{+} 1^{+}$mast cells were colocalized with GST (Figure 3E). Preferential targeting of His-tagged HRF-2CA to IgE ${ }^{+} \mathrm{mMCP}^{+}{ }^{+}$ mast cells was also observed using anti-His tag $(64.0 \% \pm 2.69 \%)$ (data not shown). These results suggest that HRF inhibitors target mainly mucosal mast cells, although their inhibitory effects on basophils might be contributory.

Intestinal epithelial cells, fibroblasts, and various immune cells secrete HRF. Immunofluorescence microscopy analysis was performed to localize HRF in jejunum, the most proximal intestinal segment where diarrhea began in this model of food allergy. HRF staining was detected both inside and outside of nuclei of intestinal epithelial cells, CD $45^{+}$hematopoietic cells, and CD45 ${ }^{-}$stromal cells in lamina propria (Supplemental Figure 5, A and B). Among hematopoietic cells, $\mathrm{IgE}^{+}$cells were surrounded by HRF (Supplemental Figure 5C) and Siglec $\mathrm{F}^{+}$cells with bilobed nuclei were costained with HRF (Supplemental Figure 5D). Moreover, CD63 ${ }^{+}$ cells, most of which were reported to be eosinophils in the steadystate lamina propria (27), were also HRF positive (Supplemental Figure 5E), suggesting that the eosinophils are HRF producers. Large cells with vesicular CD63 expression appeared after food allergy induction and were negative for intracellular HRF. These may be mast cells. In contrast, $\mathrm{CD}^{+}$and $\mathrm{B} 22 \mathrm{O}^{+}$cells were barely positive for HRF (data not shown).

We then investigated which cell types secrete HRF in in vitro cultures. We tested intestinal epithelial cells, fibroblasts, and various immune cells. CMT-93 murine intestinal epithelial cells 
A
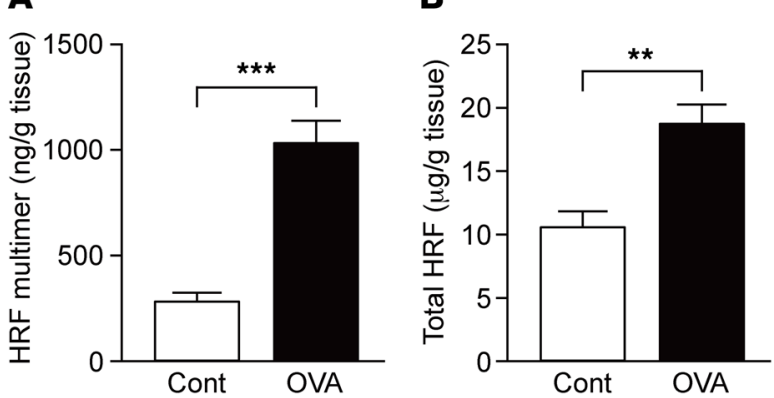

D
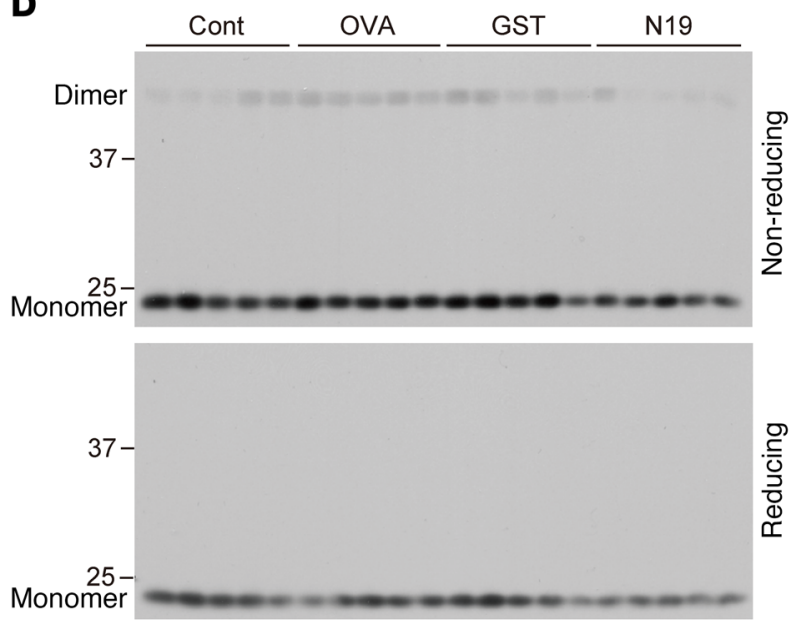

E

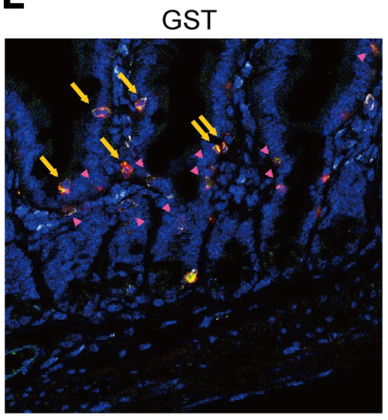

GST (or GST-N19)

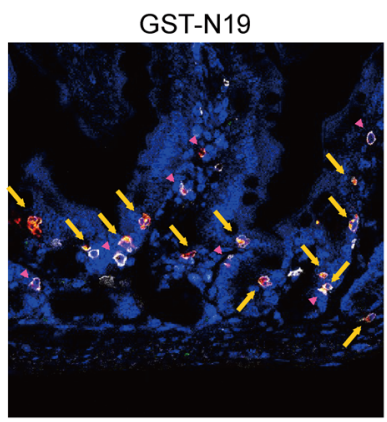

MMCP-1 IgE DAP|

\section{C}

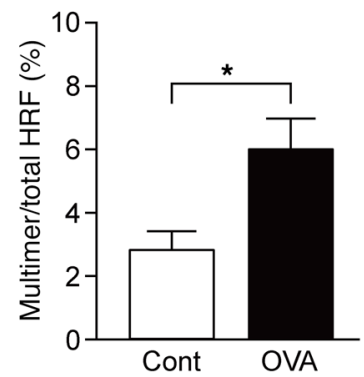

\section{展}
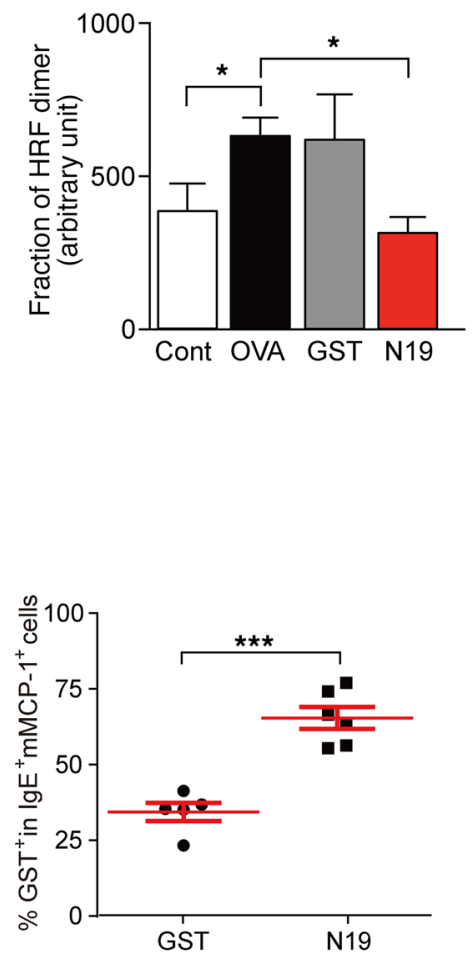

Figure 3. HRF dimer is increased in allergic mice, and GST-N19 preferentially targets mast cells in jejunum. (A and $\mathbf{C}$ ) HRF multimers in the jejunum of nonsensitized/OVA-challenged (Cont, $n$ =6) or OVA-sensitized/OVA-challenged (OVA, $n=5$ ) mice were quantified by a newly developed ELISA. (B) HRF was also measured by ELISA. (D) HRF dimer and monomer were quantified by Western blotting in control (Cont) and food allergic mice treated without (OVA) or with GST (CST) or GST-N19 (N19) ( $n=5$ for each group). (E) Allergic diarrhea was induced by 6 challenges. On the day of the seventh challenge, the mice were gavaged with $0.1 \mathrm{mg}$ of CST or CST-N19. One hour later, mice were sacrificed without OVA challenge. Jejunum tissues were stained for GST, IgE, and mMCP-1 before confocal microscopy. $n=5$ for CST; $n=6$ for GST-N19. Arrows indicate cells positive for GST or GST-N19 as well as IgE and mMCP-1. The right graph shows quantitation. ${ }^{*} P<0.05 ;{ }^{* *} P<0.01 ;{ }^{* * *} P<0.001$, Student's $t$ test. Scale bars: $50 \mu \mathrm{m}$. constitutively secreted a considerable amount of HRF monomer and stimulation with Th2 cytokines (IL-4, IL-5, and IL-13), and proinflammatory cytokines (IL-1 $\beta$, IL-6, and TNF) strongly induced HRF secretion (Figure 4A and Supplemental Figure 6A). In contrast, epithelial-derived Th2-promoting cytokines (TSLP, IL-25, and IL-33) failed to induce HRF secretion in CMT-93 cells. Fibroblasts including primary intestinal fibroblasts from newborn mice and NIH/3T3 cells also secreted HRF constitutively (Figure $4 \mathrm{~B}$ and data not shown), and stimulation with Th2 cytokines (IL-4 and IL-5), IL-1 $\beta$, and epithelial-derived cytokines, particularly IL-33, enhanced HRF secretion. IL-9 rather suppressed HRF secretion (data not shown). Consistent with results shown by imaging analysis, bone marrow-derived eosinophils produced both HRF monomer and dimer constitutively (Supplemental Figure 6B). Stimulation with IL-33 and TSLP increased HRF multimer in eosinophils. Murine RAW267.4 and human U937 macrophage lines also secreted HRF constitutively (Figure 4C and Supplemen- tal Figure 6A). IL-4, IL-5, IL-13, and IL-25 increased HRF dimer in RAW267.4 cells, while IL-1 $\beta$, IL-6, and TNF increased the secretion of HRF monomer (Figure $4 \mathrm{C}$ ). Both $\mathrm{T}$ and $\mathrm{B}$ cells secreted modest amounts of HRF, which were not affected significantly by the cytokines (Supplemental Figure 6C). In contrast, treatment of dendritic cells, bone marrow-derived macrophages, and mast cells with various classes of stimulants increased the expression of intracellular HRF, but failed to secrete significant amounts of HRF (data not shown). Thus, HRF could be secreted by various cell types present in the small intestine. Taking cell numbers into account, intestinal epithelial cells and fibroblasts seem to be the major cellular sources of secreted HRF, which dramatically increases in response to Th2 and proinflammatory cytokines.

$H R F$ inhibitors ameliorate the severity of food allergy in a therapeutic context. We next tested whether HRF inhibitors modulate food allergy after diarrhea has begun. To this end, mice were divided into 2 cohorts after OVA-sensitized mice showed 

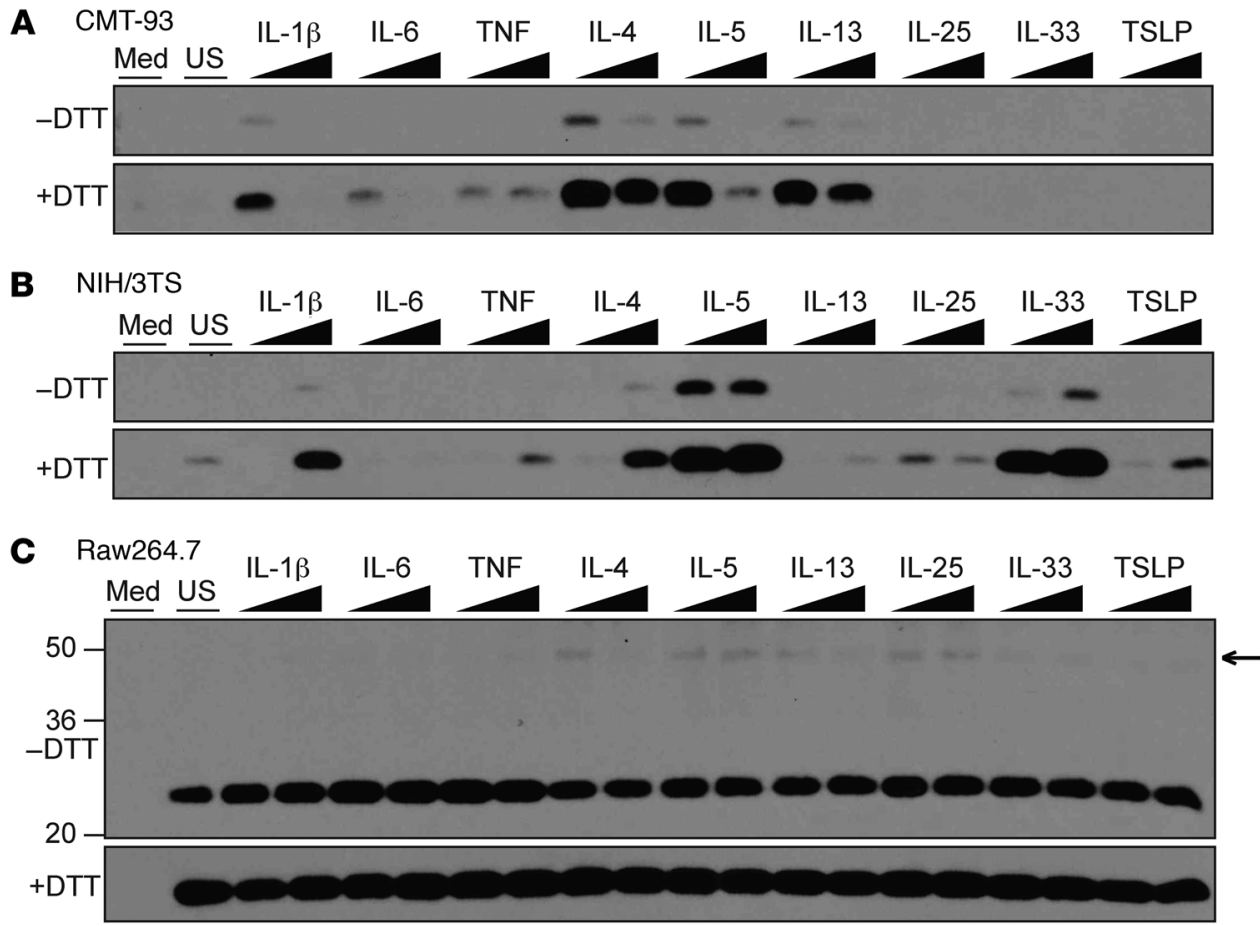

Figure 4. HRF is secreted by various cells. CMT-93 murine intestinal epithelial cells (A), NIH/3T3 murine fibroblasts, (B) and Raw264.7 murine macrophages (C) were stimulated overnight with low $(20 \mathrm{ng} / \mathrm{ml})$ and high $(100 \mathrm{ng} / \mathrm{ml})$ concentrations of various cytokines. HRF secreted into culture supernatants was detected by Western blotting. Med, mediume only; US, supernatant from unstimulated cells. -DTT and +DTT indicate SDS gels were run under nonreducing and reducing conditions, respectively. Gel portions containing the $23 \mathrm{kDa}$ HRF monomer are shown, except that a larger portion of the nonreducing gel is shown in panel C. Arrow indicates the position of HRF dimer, which disappeared under reducing conditions. diarrhea by the seventh challenge of OVA. One cohort of mice were i.v. treated twice with PBS followed by OVA challenge, and the other were i.v. treated twice with $30 \mu \mathrm{g}$ of HRF-2CA followed by OVA challenge (Figure 5A). Clinical scores were significantly reduced by HRF-2CA (Figure $5 \mathrm{~B}$ ). Therapeutic treatment of HRF inhibitor did not reduce total IgE, IgG1 (Figure $5 \mathrm{C}$ ), or mucosal mast cells (Figure 5E). However, HRF inhibitors modestly but significantly reduced serum mMCP-1 levels (Figure 5D), suggesting that the activation, but not the growth or differentiation, state of mucosal mast cells can be inhibited by HRF inhibitor after the allergic state is established. Similar therapeutic effects were observed by synthetic N19 peptide (Figure 5F) or GST-N19 (data not shown). These results collectively demonstrate that therapeutic administration of HRF inhibitors reduces the severity of food allergy.

HRF-reactive immunoglobulins during OIT in human food allergy patients. We next investigated whether $\mathrm{HRF}$ is involved in human food allergy. Similar to mouse food allergy results, eggallergy patients had plasma levels of HRF similar to those of normal individuals (data not shown) and significantly higher levels of HRF-reactive IgE and IgG levels (Figure 6, B and E). We analyzed full time-course samples from patients who were treated with rush OIT (28) to attain a level of desensitization without showing unbearable adverse effects, such as refractory gastroenteritis and/or severe anaphylaxis (Figure 6A). Plasma levels of HRF were not changed during the OIT (data not shown). However, levels of HRF-reactive IgE, IgGs, IgG1, and IgG4 were modestly reduced 1 week after the initiation of OIT (Figure 6, C and $\mathrm{F}$, and data not shown). Following 2 weeks of allergen avoidance after the end of the 12-month maintenance dosing, patients exhibited a wide range of sensitivity to egg protein upon oral challenge. Interestingly, HRF-reactive IgE levels in the patients who exhibited a low threshold (sensitive to $\leq 300 \mathrm{mg}$ of egg white protein) were significantly higher than their HRF-reactive IgE levels 1 week after the OIT initiation (Figure 6, D and H), whereas HRF-reactive IgE levels in those who exhibited higher thresholds (>300 mg of egg white protein) were not significantly different from their 1-week values (data not shown). In contrast, HRF-reactive IgGs, IgG1, and IgG4 levels after 2 weeks of allergen avoidance following the 12-month maintenance period were similar to the levels 1 week after the OIT initiation in all patients, regardless of their changes in threshold to egg protein (Figure 6G and data not shown).

Consistent with earlier studies suggesting that the initial antigen-specific IgE level is a good predictive biomarker for the prognosis of OIT (29), low egg white-specific IgE levels before OIT were predictive for sustained desensitization to egg (Figure 6J). In addition, all patients with HRF-reactive IgE above a certain level (400 counts) before OIT initiation tolerated at least 1,000 mg of egg protein after 12 months of OIT (Figure 6I). The HRF-r eactive IgE level, which showed no correlation to egg whitespecific IgE (Pearson's correlation, $r=0.12, P=0.47$ ) or to total $\operatorname{IgE}(r=0.0071, P=0.97)$ (Figure 6I and data not shown), was not only predictive for good prognosis by itself, but also synergistically predictive when combined with egg white-specific IgE, reaching a specificity of $82 \%$ and sensitivity of $85 \%$ (Figure 6J). These results suggest that HRF-reactive IgE might serve as an independent predictive biomarker for OIT outcome.

$H R F$-reactive IgE is sustained at low levels in murine OIT models. We next investigated when HRF-reactive IgE levels increased during food allergy induction. Similar levels of HRF-reactive IgE were found before and after OVA challenges in nonsensitized mice and before OVA challenges in sensitized mice (Figure 7A). Interestingly, HRF-reactive IgE levels increased by OVA challenges in OVA-sensitized mice, and HRF inhibitor (HRF-2CA) did not affect HRF-reactive IgE levels. In contrast, OVA-specific IgE lev- 
A

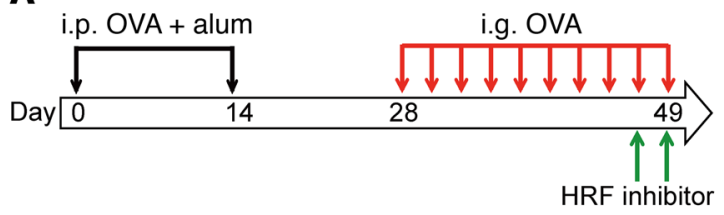

B

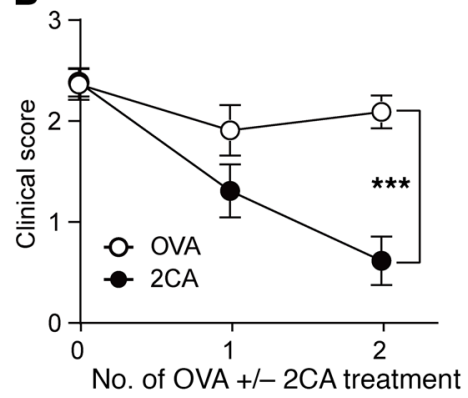

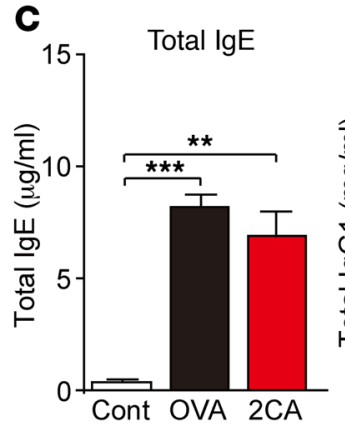

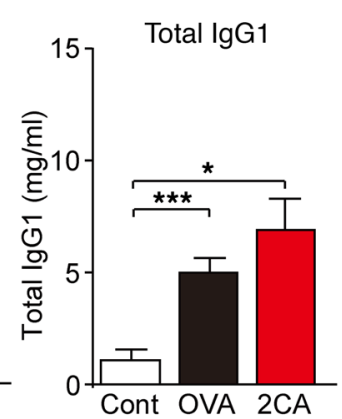

D

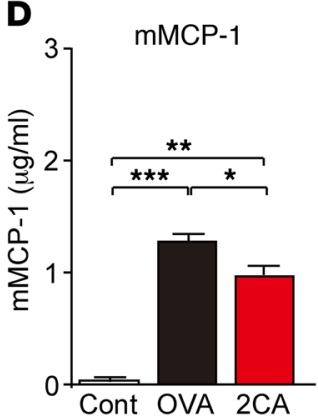

E

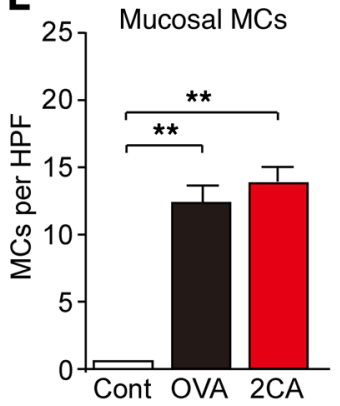

$\mathbf{F}$

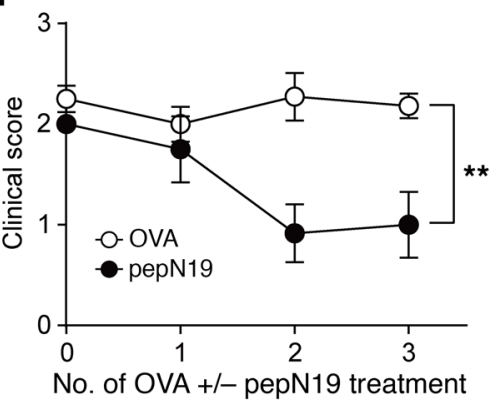

Figure 5. Therapeutic treatment with HRF inhibitor ameliorates the severity of food allergy. Mice were sensitized and i.g. challenged with OVA. By the seventh OVA challenge, all the mice suffered diarrhea. These mice were pretreated i.v. with $30 \mu \mathrm{g}$ of HRF-2CA (2CA) or PBS (OVA) before being challenged with OVA 2 more times. (A) Procedure schematic. (B) Diarrhea severity. $n=11$ for OVA; $n=13$ for HRF-2CA; $n=4$ for nonsensitized control (not shown); pooled data of 2 independent experiments) ${ }^{* * *} P<0.001$, 2-way ANOVA. (C) Total and OVA-specific IgE and IgG1 concentration in sera were measured by ELISA. (D) mMCP-1 in sera was measured by ELISA. (E) Sections of jejunum were stained with chloroacetate esterase. (C-E) ${ }^{*} P<0.05 ;{ }^{* *} P<0.01$; ${ }^{* * *} P<0.001$, Student's $t$ test. (F) Similar i.v. therapeutic treatment of allergic mice was conducted with $30 \mu$ g of synthetic N19 peptide. $n=12$ for OVA; $n=12$ for pepN19, pooled data of 2 independent experiments. ${ }^{* *} P<0.001,2$-way ANOVA.

els increased before OVA challenges in OVA-sensitized mice and failed to significantly increase by OVA challenges. These results indicate that, while anti-OVA IgE Ab was generated by sensitization, HRF-reactive IgE levels increased under the Th2 and inflammatory cytokine-rich environment during the elicitation phase, similar to jejunal HRF levels (Figure 3, A-D). In contrast, HRFreactive IgG levels, which peaked by the ninth OVA challenge (Figure 1E), were reduced after eleven OVA challenges in both nonsensitized and sensitized mice (Figure 7A). The reduction of HRF-reactive IgG levels was not affected by HRF-2CA. However, OVA-specific IgG levels increased by sensitization and were not significantly altered by OVA challenges, similarly to OVA-specific IgE levels. Thus, HRF-reactive IgG and HRF-reactive IgE levels were altered in different kinetics.

We then tested the possibility provoked by the above human data that the level of HRF-reactive IgE is kept low in a desensitized state, using 2 murine models. In a classic tolerance model (30), 5 daily oral administrations of $1 \mathrm{mg}$ OVA from day -11 to day -7 (OIT) prevented the OVA-induced development of allergic diarrhea in OVAsensitized mice (Figure 7B). In contrast, without oral pretreatment, all OVA-sensitized mice developed allergic diarrhea. HRF-reactive IgE levels were significantly reduced in tolerance-induced mice compared with nontolerant and nonsensitized mice (Figure 7C).
Finally, we monitored HRF-reactive IgE and HRF-reactive IgG in another desensitization model, which mimicked the human OIT more faithfully. OVA-sensitized mice were first rendered allergic by OVA challenges and then received hourly increasing amounts of OVA for 3 consecutive days (OIT) after a resting period (Figure 7D). OIT made $80 \%$ of OVA-sensitized/OVA-challenged mice desensitized to the final gavage dose of $60 \mathrm{mg}$ of OVA, whereas non-OIT treatment resulted in diarrhea reactive to $60 \mathrm{mg}$ of OVA in $75 \%$ of OVA-sensitized/OVA-challenged mice. Similar to human OIT results, mouse OIT also lowered levels of HRFreactive IgE, whereas it did not change levels of OVA-specific IgE (Figure 7E). Levels of HRF-reactive IgG and OVA-specific IgG were not changed by OIT (Figure 7E). In nonsensitized mice, HRFreactive IgE and HRF-reactive IgG levels were not changed by OIT and OVA-specific IgE, and IgG levels were extremely low irrespective of OIT treatment (data not shown). These results, along with the human OIT data, indicate that the desensitized state induced by OIT is associated with low HRF-reactive IgE levels.

\section{Discussion}

Little was known about the role of HRF in food allergy before the present study. This was partly because HRF-knockout mice were embryonically lethal $(31,32)$. However, our study circumvented 
A

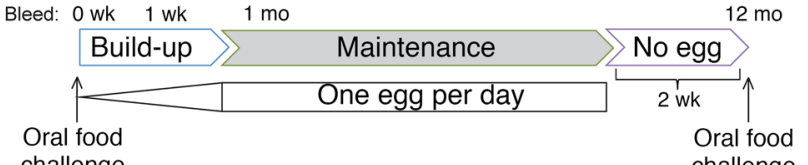

B

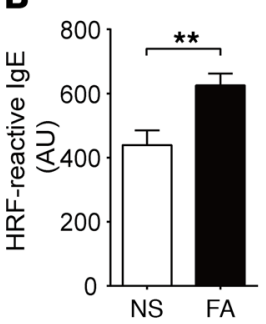

C
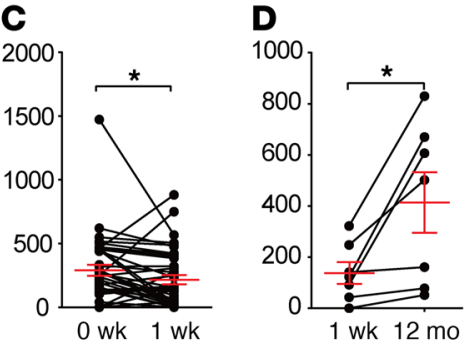

E

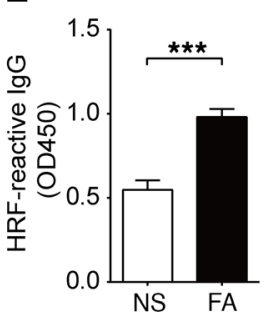

$\mathbf{F}$

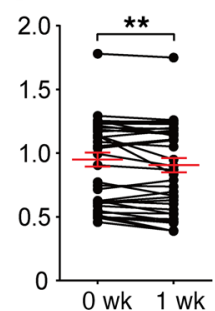

G

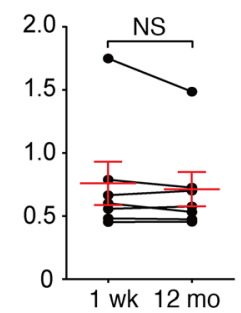

H

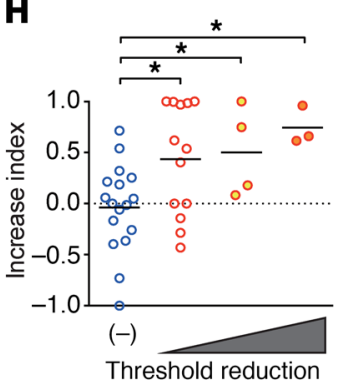

I

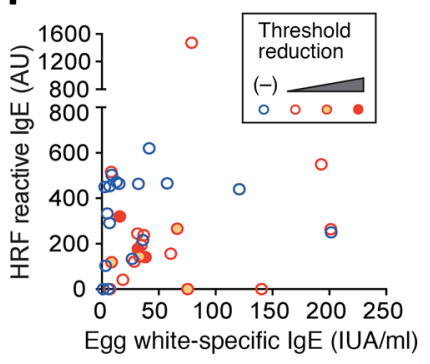

$\mathbf{J}$

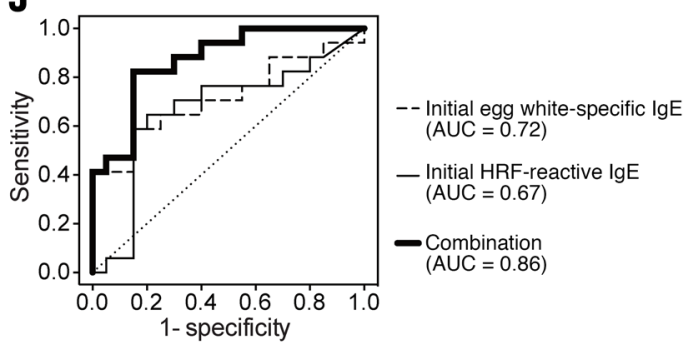

this obstacle by using HRF inhibitors that blocked HRF-IgE interactions, which clearly suppressed food allergy in mice when administered prophylactically and therapeutically. Despite the lack of HRF-related food allergy studies in the past, an interesting study was reported (33) long before the molecular identity of HRF was revealed (16): patients with food allergy and atopic dermatitis (AD), but not patients with $\mathrm{AD}$ without food allergy, were found to have higher rates of spontaneous release of histamine from basophils than normal subjects; this histamine-releasing activity declined when patients avoided the offending foods for an extended period. While those old data cannot be interpreted with regard to $\mathrm{HRF}$, it will be interesting to study the role of $\mathrm{HRF}$ in $\mathrm{AD}$.
Figure 6. Reduced HRF-reactive IgE levels correlate with effective OIT outcomes in food allergy. (A) Schematic of a rush OIT. (B-C) HRF-reactive IgE (B-D) and IgG (E-C) levels were measured by ELISA before, 1 week after, and 12 months after OIT initiation in normal subjects (NS) and food allergy patients (FA). (B and E) Data represent all patients. $n=9$ for normal subjects; $n=42$ for food allergy patients. Samples from 1 patient were not available due to sample shortage. (C and $\mathbf{F}$ ) Data represent patients who underwent the full OIT regimen $(n=37)$. ( $\mathbf{D}$ and $\mathbf{G})$ Data represent patients showing considerable and severe reductions in threshold $(n=7)$. ${ }^{*} P<0.05,{ }^{* *} P<0.01,{ }^{* *} P<0.001$; Analyzed by Student's $t$ test (B and $\mathbf{E}$ ) and paired $t$ test (C, D, F, and $\mathbf{G})$. (H) HRF-reactive IgE increase indices (Supplemental Methods) were compared among the 4 cohorts by ANOVA with Holm-Šídák's multiple comparison. $n=17$ for stable desensitization. $n=13,4$ and 3 for patients with reduced threshold to 1000,300 , and 100 mg of egg-white powder, respectively. (I) Distribution of HRF-reactive IgE and egg white-specific IgE levels before OIT in egg-allergy patients $(n=37)$. (J) Comparison of receiver operating characteristic (ROC) curves showing the performances of initial HRF-reactive IgE (solid line), initial egg whitespecific IgE (broken line), and a combination of both factors (bold line) in predicting prolonged desensitization at 12 months after OIT.

Although HRF inhibitors' effects were limited to the elicitation phase in our model, this study does not exclude the possibility that HRF might play a role in the sensitization phase in human food allergy. Sensitization with allergen in the presence of alum skews the immune response to a Th2 type so strongly that potential involvement of $\mathrm{HRF}$, which might operate in a more physiological sensitization condition, might not present itself under the employed condition. As the role of antigennonspecific IgE in sensitization was shown in contact sensitivity (34) and the IL-4R $\alpha$ 709F model of food allergy (35) by Hans Oettgen and associates, further study is warranted on the role of HRF in the sensitization phase using a different model.

HRF dimer can exert its action via HRF-reactive IgE bound to FceRI, leading to the activation of mast cells and basophils (19). HRF-reactive IgE levels increased during the elicitation phase, unlike antigen-specific IgE levels that increased by sensitization. Both antigen and HRF dimer can activate IgE-sensitized mast cells independently. Although the prevailing view in the field indicates that mast cell activation through FceRI is due to receptor crosslinking with $\operatorname{IgE}$ and antigen (36), we propose that intestinal mast cells in food allergic mice are synergistically activated by OVA and HRF dimer (or possibly HRF oligomers). This synergistic mast cell activation by antigen and HRF could also be found in asthma, AD, and other allergic diseases. Intestinal mast cells from nonsensitized mice were activated with anti-IgE, but not with OVA, indicating that a considerable proportion of the FceRI molecules expressed on these mast cells were occupied by nonspecific IgE. In contrast, mast cells from food-allergic mice were activated with OVA, indicating that a substantial proportion of the FceRI molecules on these cells were occupied by OVAspecific IgE molecules. Since some of both OVA-specific and nonspecific IgE molecules should interact with HRF (19), activation of mast cells in food-allergic mice could be triggered by both OVA and HRF dimer/multimers. Interestingly, successful OIT promoted reductions in HRF-reactive IgE without significantly affecting OVA-specific IgE levels. Therefore, reduced HRFreactive IgE levels likely contributed to OIT-induced suppression of mast cell activation. Furthermore, our study suggests that pre- 
A
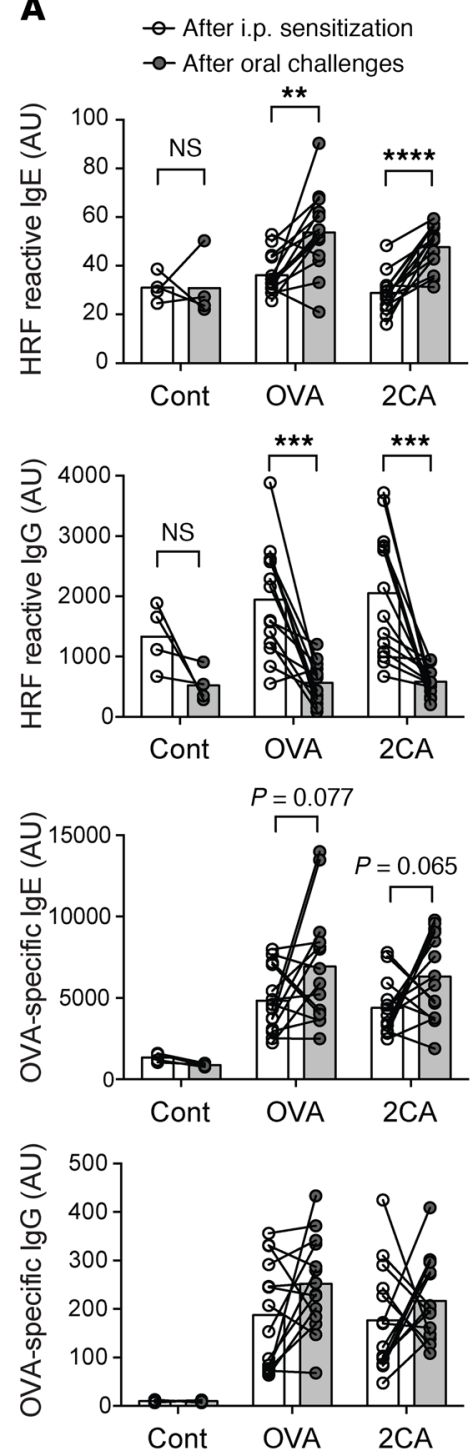

B
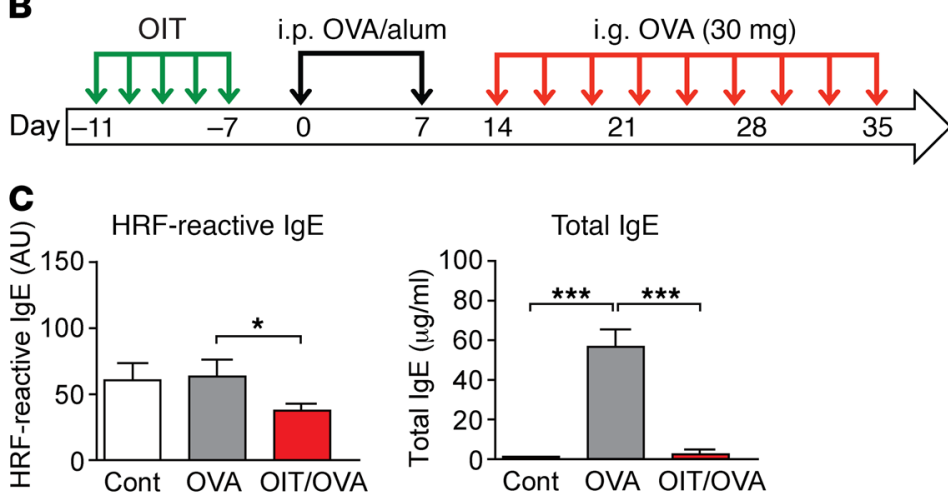

D
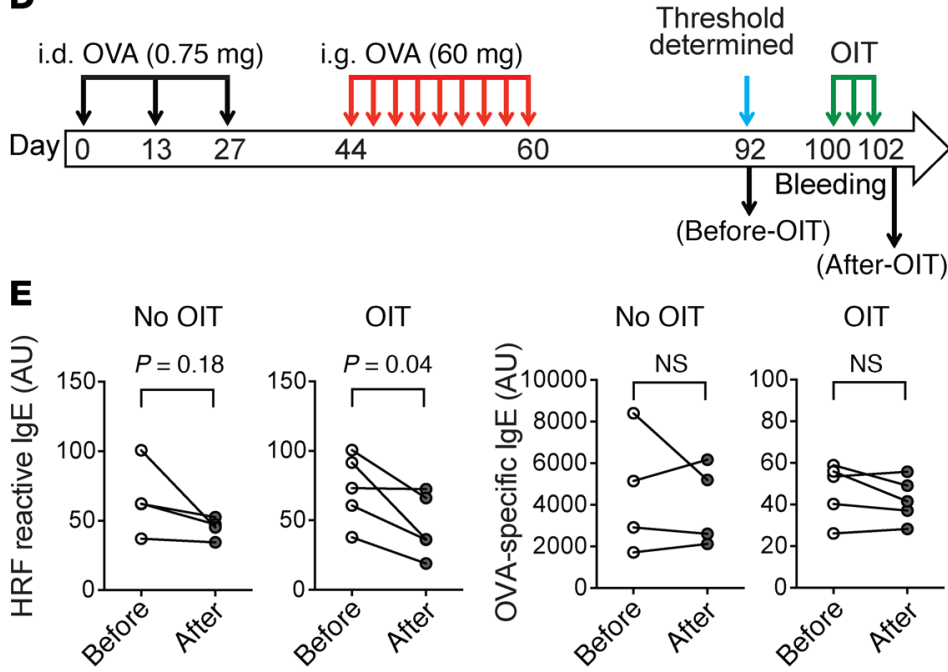

(After-OIT)
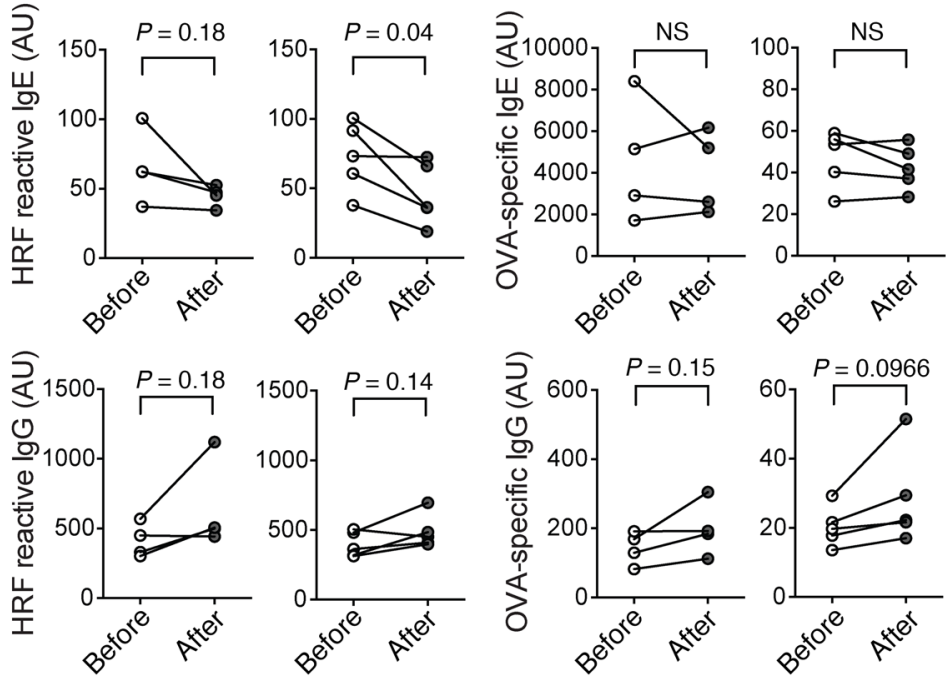

Figure 7. HRF-reactive IgE levels are low in desensitized mice. (A) Mice were unsensitized (Cont) or i.p. sensitized as described in the Figure 1 legend and i.g. challenged with OVA with nonpretreated (OVA) or i.g. pretreated HRF-2CA (2CA). HRF-reactive IgE and IgG and OVA-specific IgE and IgG after sensitization (before OVA gavages) and after OVA gavages were measured. $n=4$ for nonsensitized group; $n=14$ for OVA group; $n=14$ for 2 CA group, pooled data of 2 independent experiments. ${ }^{* *} P<0.01 ;{ }^{* *} P<0.001 ;{ }^{* * *} P<0.0001$, paired $t$ test. (B and C) Five daily oral administrations of 1 mg 0 VA from day -11 to day -7 were followed by sensitization with OVA and then by OVA challenges in mice. Without oral pretreatment, which prevented diarrhea, all OVA-sensitized mice developed allergic diarrhea. At the end of the experiment, HRF-reactive IgE and total lgE were quantified. $n=5$ for nonsensitized group; $n=10$ for OVA group; $n=9$ for OIT/OVA group, pooled data of 2 independent experiments. ${ }^{*} P<0.05$, Student's $t$ test (C). (D and E) Mice were intradermally sensitized with OVA followed by oral OVA challenges. All mice showed diarrhea. Then some mice received hourly increasing amounts of OVA for 3 consecutive days (OIT). Levels of HRF-reactive IgE and IgG as well as OVA-specific IgE and IgG were quantified by ELISA before and after OIT ( $n=4$ for no OIT group and $n=5$ for OIT group). Statistics by paired $t$ test.

OIT levels of HRF-reactive IgE might be a useful biomarker to predict the outcome of OIT. Therefore, future study is warranted on how HRF-reactive IgE levels are regulated.

It remains unknown what triggers an allergic reaction in response to ingested food. Probably, epithelial-derived cytokines such as TSLP, IL-25, and IL-33 play an important role in initiating a type 2 immune response in allergic diseases, including food allergy $(6,37-40)$. Using mice sensitized epicutaneously with OVA followed by oral challenge with OVA, basophil-depleted or TSLP receptor-deficient mice were shown to not produce OVA-specific IgE and to be protected from oral challenge-induced anaphylaxis $(21,41)$. Epicutaneous sensitization of mice with OVA and TSLP, followed by oral challenges with OVA, resulted in IL-25dependent diarrhea and anaphylaxis (42). IL-33-deficient mice produced normal levels of OVA-specific IgE, whereas IL-33deficient and soluble IL-33 receptor-treated mice were protected 
from anaphylaxis (41). Thus, basophils and TSLP have pivotal roles in Th2 development in the skin during the sensitization phase of food allergy. In contrast, while IL-33 is dispensable for promoting cutaneous antigen sensitization, it is essential for inducing IgEdependent anaphylaxis in the gut $(21,41)$. Transgenic overexpression of IL-25 promoted the susceptibility to food allergy, whereas IL-25 receptor-deficient (IL-17RB-deficient) mice were resistant to food allergy induction. CD $4^{+} \mathrm{Th} 2$ cells and type 2 innate lymphoid cells (ILC2s) promote the susceptibility to food allergy by interacting with each other to produce a large amount of IL-13 in response to IL-25 (43). IL-13 primarily produced by ILC2s promotes food allergy by inducing goblet cell hyperplasia and increasing intestinal permeability. In contrast, HRF secretion by intestinal epithelial cells and fibroblasts was enhanced by Th2 cytokines (IL-4, IL-5, and IL-13) and proinflammatory cytokines (IL-1 $\beta$ and TNF), and epithelial-derived IL-33 enhanced HRF secretion in fibroblasts; Th2 and epithelial cytokines stimulated HRF dimer secretion. Thus, we propose a scenario in which HRF amplifies Th2 inflammation after initial inflammation is triggered by allergenmediated activation of ILC2s and Th2 cells (Supplemental Figure 7). These cytokine-regulated highly orchestrated events lead to IgEdependent mast cell (and basophil) activation via FceRI.

Clinical studies are underway to investigate the efficacy of immunotherapy in food allergy. Allergen-specific sublingual, oral, and epicutaneous immunotherapies were reported to successfully treat some patients $(3,4)$. Our results with eggallergy patients suggest the pathological role for HRF in human egg allergy: first, HRF-reactive IgE and HRF-reactive IgG were higher in egg-allergy patients than normal controls, and OIT caused their reduction. Second, high HRF-reactive IgE levels before OIT initiation, similar to low levels of egg whitespecific IgE, were predictive of OIT success. Although the good correlation of high initial HRF-reactive IgE levels with positive outcomes of OIT might be counterintuitive, the disease of such patients might be highly dependent on HRF-mediated allergic inflammation. Third, HRF-reactive IgE levels became higher only with patients who exhibited high sensitivity to egg protein after OIT. Two murine OIT models also supported this last point by showing that successful OIT keeps HRF-reactive IgE levels low. These results, if replicated in other clinical trials, will encourage patients with high levels of HRF-reactive IgE and low levels of egg white-specific IgE to go through a long process of OIT with a high prospect of success. Further research is highly warranted to investigate whether HRF-reactive $\operatorname{IgE}$ could serve as a biomarker for successful immunotherapy of patients with egg allergy or other food allergies.

In conclusion, this study demonstrates that HRF plays a type 2 inflammation-amplifying role during the elicitation phase of food allergy in a well-characterized murine model. HRF dimer/multimers increased in their local concentration relative to HRF monomer in the small intestine. HRF dimer could enhance IgE-mediated activation of mucosal mast cells in the small intestine of allergic mice. As further evidence for $\mathrm{HRF} /$ IgE-mediated mast cell activation via FceRI, blockade of HRFIgE interactions inhibits antigen- or HRF dimer-induced mast cell activation in vivo and ex vivo in WT, but not FceRI-deficient, mice. Therefore, this study identifies HRF as a prophylactic and therapeutic target in food allergy and implies HRF-reactive IgE as a biomarker for the prognosis of OIT.

\section{Methods}

Egg-allergy patients and OIT. Patients (43 patients, 37 males and 6 females, average age $7.3 \pm 2.1$ years) were confirmed to have hen's egg allergy by oral challenge test at the enrollment and received rush OIT that enabled 38 participants to eat about 1 egg $(60 \mathrm{~g})$ and $1 \mathrm{~g}$ of egg white powder per day without unbearable adverse effects, such as refractory gastroenteritis and/or severe anaphylaxis (28). These patients kept eating this amount of egg for 12 months. Then, after 2 weeks of egg avoidance, the patients took an oral challenge test, results of which were used to stratify patients into 4 groups, with no (threshold, $\geq 1000 \mathrm{mg}$ egg protein), mild (300-1000 mg), considerable (100$300 \mathrm{mg}$ ), and severe ( $\leq 100 \mathrm{mg}$ ) reductions in threshold. HRF, HRFreactive IgE, and HRF-reactive IgG levels in plasma collected before, 1 week after, and 12 months after OIT initiation were measured by ELISA. Due to sample shortage, some samples from 1 patient were not available. Therefore, 42 initial samples and 37 time-course samples were analyzed. This study has been registered at UMIN Clinical Trials Registry (UMIN000003943).

Mice. BALB/c mice were bred in the animal facility of RIKEN IMS. Frozen zygotes of $\mathrm{FceRIa}^{-/-}$mice were donated by Takashi Saito (RIKEN IMS) and bred at RIKEN IMS. FcgRIIB ${ }^{-/-}$mice provided by Toshiyuki Takai (Tohoku University, Sendai, Japan) were also bred at RIKEN IMS. Food allergy experiments with Enpp $3^{-/-}$mice were conducted at Osaka University. All genetically modified mice were backcrossed to a $\mathrm{BALB} / \mathrm{c}$ background for more than 10 generations.

HRF inhibitors. GST and GST-N19 were purified using glutathione-agarose (Sigma-Aldrich). HRF-2CA-His ${ }_{6}$ expressed by pET$24 \mathrm{a}(+)$ plasmid was purified using ProBond resin (Invitrogen). All recombinants were further purified by Sephacryl S-100 and dialyzed against PBS. N19 peptide was synthesized by Eurofins Genomics.

IgE-mediated experimental food allergy and OIT. Mice were i.p. sensitized with OVA $(50 \mu \mathrm{g} / \mathrm{mouse})$ plus alum on days 0 and 14 . From day 28 , mice were i.g. challenged with OVA ( 25 or $50 \mathrm{mg}$ ) or PBS (control) 3 times a week. Before each challenge, mice were starved for 3 hours, then i.g. pretreated with GST, GST-N19 (100 $\mu \mathrm{g} / \mathrm{mouse})$, synthetic N19 peptide, or HRF-2CA. The development of diarrhea was monitored for 90 minutes after OVA challenge. Diarrhea severity (30) was based on states of stool: solid (score 0), funicular (score 1), slurry (score 2), and watery (score 3).

In a classical tolerance model, 5 daily oral administrations of $1 \mathrm{mg}$ OVA from day -11 to day -7 were performed before i.p. OVA/alum sensitization on days 0 and 7 and followed by i.g. OVA (30 mg) gavages on days $14,21,28$, and 35 . In another OIT model, mice were intradermally sensitized with OVA (750 $\mu \mathrm{g})$ on days 0,13 , and 27 and followed by 9 oral OVA $(60 \mathrm{mg}$ ) challenges from day 44 to day 60 to induce diarrhea in all mice. Then mice were i.g. treated with hourly increasing amounts of OVA on day 100 (0.1, 0.2, $0.5 \mathrm{mg})$, on day 101 (1, 2, $5 \mathrm{mg}$ ), and on day 102 (10, 20, $60 \mathrm{mg})$.

Measurement of parameters of food allergy. For histologic analysis, jejunal tissue was fixed in $10 \%$ formalin. Mast cells in jejunal sections were detected by chloroacetate esterase staining. IgE, IgG, IgG1, IgG2a, mMCP-1, HRF, HRF-reactive IgE, and HRF-reactive IgG 24 hours after the last OVA challenge were measured by ELISA (see Supplemental Methods). Cytokine mRNAs in jejunum were quantified by qRT-PCR. 
Preparation of lamina propria cells. Twenty-four hours after the last OVA challenge, mice were sacrificed and small intestines $(0.5-15 \mathrm{~cm}$ from stomach) were collected. The intestinal tissues were opened longitudinally and washed with HBSS. The washed tissues were incubated with $5 \mathrm{mM}$ EDTA-HBSS for 30 minutes at $4^{\circ} \mathrm{C}$, transferred to $30 \mathrm{ml}$ HBSS in $50 \mathrm{ml}$ tubes, and vigorously shaken to remove intestinal epithelial cells. The tissues were then washed with $10 \mathrm{ml}$ of 10\% FCS RPMI medium. Tissues were cut into tiny pieces and incubated with $2.4 \mathrm{mg} /$ $\mathrm{ml}$ collagenase $\mathrm{D}$ and $0.2 \mathrm{mg} / \mathrm{ml}$ DNase for 30 minutes at $37^{\circ} \mathrm{C}$. Then cells were washed, resuspended in $44 \%$ Percoll solution, and slowly loaded on the top of $67 \%$ Percoll solution. The cells were centrifuged (650 x $g, 20$ minutes), and lymphocyte fractions were collected and used for detection of MMC9s ( Lin $^{-}$-Kit ${ }^{+}$ST2 $2^{+}$IL-17RB-Integrin $\beta 7^{10}$ ).

Flow cytometry to quantify MMC9s. The cells were first incubated with $2.4 \mathrm{G} 2$ for 15 minutes at $4^{\circ} \mathrm{C}$ and further incubated with the following Abs: PE-anti- $\beta 7$ integrin $\mathrm{mAb}$ (clone FIB504), PerCP/Cy5.5anti-CD4 mAb (clone RM4-5), PerCP/Cy5.5-anti-CD8 mAb (clone 53-6.7), PerCP/Cy5.5-anti-CD11b mAb (clone M1/70), PerCP/Cy5.5anti-CD11c mAb (clone N418), PerCP/Cy5.5-anti-B220 mAb (RA36B2), PerCP/Cy5.5-anti-Ly-6G/Ly-6C mAb (clone RB6-8C5), PerCP/ Cy5.5-anti-CD335 mAb (clone 29A1.4), Alexa Fluor 647-anti-IL-17RB (clone 9B10), APC/Cy7-anti-CD117 (clone 2B8), and biotin-anti-ST2 (clone DIH9) (BioLegend) for 30 minutes at $4^{\circ} \mathrm{C}$. After washing, cells were incubated with SA-BV421 (BioLegend) for 30 minutes at $4^{\circ} \mathrm{C}$. The MMC9 population was identified using BD FACSCanto. Data were analyzed using FlowJo software.

Exvivo MCAT. Twenty-four hours after the last OVA challenge, mice were sacrificed and small intestines $(0.5-15 \mathrm{~cm}$ from stomach) were collected. The intestinal tissues were opened longitudinally and washed with PBS. Then the tissues were incubated with $10 \mathrm{mM}$ EDTA-PBS for 30 minutes at $37^{\circ} \mathrm{C}$. After incubation, the tissues were transferred to 30 $\mathrm{ml} \mathrm{PBS}$ in $50 \mathrm{ml}$ tubes and vigorously shaken. After washing, the cells were resuspended in $40 \%$ Percoll solution and slowly loaded on the top of $75 \%$ Percoll solution. The cells were then centrifuged $(800 \mathrm{x} g, 20$ minutes) and lymphocytes were collected. The cells were centrifuged and resuspended in Tyrode's buffer. The following Abs were added: biotin-anti-CD4 mAb (clone RM4-5), biotin-anti-CD8 mAb (clone 53-6.7), biotin-anti-B220 mAb (clone RA3-6B2), biotin-anti-CD326 mAb (clone G8.8), PE-anti-CD117 (clone 2B8), and Alexa Fluor 647anti-LAMP-1 mAb (clone 1D4B) (BioLegend). Cells were then stimulated with OVA, anti-IgE (clone RME-1, BioLegend) and recombinant dimeric murine $\mathrm{HRF}$ for 15 minutes at $37^{\circ} \mathrm{C}$. After activation, cells were washed and incubated with FITC-streptavidin (catalog 405202, BioLegend) for 30 minutes at $4^{\circ} \mathrm{C}$. LAMP- 1 expression was detected using FACSCalibur. Data were analyzed using FlowJo software.

ELISA to quantify HRF dimer/multimers. Anti-HRF mAb (clone 2A3, Abnova) was immobilized in a 96-well plate (Corning) at $4^{\circ} \mathrm{C}$ overnight. The plate was blocked with $5 \%$ skim milk and $10 \%$ FCS. After washing, plates were incubated with $50 \mu \mathrm{l} /$ well of a standard or sample at room temperature (RT) for 2 hours on a rotating table. After washing, 1 hour incubation with $50 \mu \mathrm{l}$ of $1 \mu \mathrm{g} / \mathrm{ml}$ biotinylated mAb $2 \mathrm{~A} 3$ followed. Then the plates were incubated with $50 \mu \mathrm{l}$ of 1:1000 diluted avidin-HRP (BioLegend) at RT for 30 minutes. After extensive washing, $50 \mu \mathrm{l} /$ well of ECL Pro (PerkinElmer) was added to the plate and luminescence was measured in a luminescent microplate reader.

Western blot analysis of HRF in various cells. The following cell lines were cultured in DMEM plus 10\% FCS: CMT-93 mouse intestinal epi- thelial cell line, NIH/3T3 mouse fibroblast line, raw 264.7 mouse and U-937 human macrophage lines, BEAS-2B human bronchial epithelial cell line, and 293T human embryonic kidney cell line. Caco-2 human epithelial cell line was cultured in DMEM with 20\% FCS, and U-937 cells were cultured in RPMI 1640 plus 10\% FCS. All cell lines were obtained from ATCC, except for NIH/3T3, which was provided by S.A. Aaronson, NIH). Fibroblast-like stromal cells were cultured from small intestines of BALB/c mice in DMEM/10\% FBS. CD4 ${ }^{+} \mathrm{T}$ and $\mathrm{B}$ cells were purified from mouse spleens using BD IMag Mouse CD4 and B cells Enrichment Set-DM. Low-density bone marrow cells were cultured in the presence of $100 \mathrm{ng} / \mathrm{ml}$ of stem cell factor (PeproTech) and $100 \mathrm{ng} / \mathrm{ml}$ of FLT-3 ligand (R\&D Systems) for 4 days and with 10 $\mathrm{ng} / \mathrm{ml}$ of IL-5 (R\&D Systems) thereafter for 10 days to obtain bone marrow-derived eosinophils (44). Bone marrow cells were cultured in the presence of $20 \mathrm{ng} / \mathrm{ml}$ of granulocyte-macrophage CSF (GM-CSF) for 6 days, and adherent cells were collected as bone marrow-derived macrophages. Nonadherent cells were further cultured with $5 \mathrm{ng} /$ $\mathrm{ml} \mathrm{GM-CSF}$, and the nonadherent cells were collected as bone marrow-derived dendritic cells. Bone marrow cells were cultured in IL-3containing medium for 4 to 6 weeks to generate more than $95 \%$ c-Kit ${ }^{+} \mathrm{Fc} \varepsilon \mathrm{RI}^{+}$mast cells. All cells were stimulated with $100 \mathrm{ng} / \mathrm{ml} \mathrm{LPS}$, $20 \mathrm{ng} / \mathrm{ml}$ PMA plus $1 \mathrm{mM}$ ionomycin, and $100 \mathrm{ng} / \mathrm{ml} \mathrm{IL-13}$ in $0.1 \%$ BSA RPMI 1640 medium for 3 hours or 6 hours.

Immunofluorescence microscopy. Jejunum biopsy was fixed in $4 \%$ paraformaldehyde at $4^{\circ} \mathrm{C}$, washed in 10\%-20\% sucrose in PBS, and embedded in OCT compound (Sakura Finetek). Cyrosections $6-\mu \mathrm{m}$ thick were prepared and blocked with $2 \%$ skim milk. Immunostaining was performed with anti-HRF (rabbit polyclonal FL-172), anti-GST (rabbit polyclonal Z-5) (Santa Cruz Biotechnology Inc.), anti-IgE (clone RME-1, BioLegend; clone R35-72, BD Biosciences), anti-mMCP1 (catalog AF5146, R\&D Systems), anti-CD45 (clone 30-F11), anti-CD63 (clone NVG-2, BioLegend), and anti-Siglec F (clone E50-2440, BD Biosciences) as primary Abs, and anti-rabbit Alexa Fluor 647 (catalog A21245), anti-rat Alexa Fluor 568 (catalog A11077), and anti-sheep Alexa Fluor 555 (catalog A11015) (Thermo Fisher Scientific) as secondary Abs. Coverslips were mounted with ProLong Gold with DAPI (Thermo Fisher Scientific), and fluorescence was observed under Leica TCS SP2 confocal microscopy and Nuance Multispectral Imaging System (PerkinElmer).

Statistics. Statistical analysis was performed using Prism6 software (GraphPad). Data are presented as mean \pm SEM in all figure parts in which error bars are shown. Groups were analyzed by 2-tailed Student's $t$ test, paired $t$ test, and 2-way ANOVA, unless otherwise indicated. $P<0.05$ was considered statistically significant.

Study approval. Human studies were performed according to Declaration of Helsinki Principles. The study protocol was approved by the Ethical Review Board of Mie National Hospital and the RIKEN Yokohama Campus Ethics Committee. Written informed consent was obtained from each of the study participants and their guardians prior to participation. Animal experiments were approved by the Animal Care and Use Committee of the La Jolla Institute for Allergy and Immunology, the RIKEN Yokohama Animal Experiments Committee, and the Animal Experimentation Committee of Osaka University.

\section{Author contributions}

TA and JK designed and performed most of the experiments. NIN, TI, NS, TF, and MN conducted the OIT study. HY, MB, Yu Kawaka- 
mi, SHT, and KM performed experiments. NI and KT directed part of the study. Yuko Kawakami, and TK conceived and directed the entire project. TK wrote the manuscript with input from all coauthors.

\section{Acknowledgments}

We appreciate Kazuko Kanegae for her excellent technical help. We are grateful to Masato Kubo (RIKEN Center for Integrative Medical Sciences) for allowing TA and JK to finish this project; Toshiyuki Takai (Tohoku University) for kindly providing mice; and Yui-Hsi Wang (Cincinnati Children's Hospital) for sharing his protocol for MMC9 identification. This study was supported in part by grants from the US NIH (HL124283, AR064418, AI115534 and AI124734), the Ministry of Education, Culture, Sports, Science and Technology, Japan (25670483, 26860765, 15K10794), the Japan Research Foundation for Clinical Pharmacology, and the Nipponham Foundation for the Future of Food.

Address correspondence to: Toshiaki Kawakami, Division of Cell Biology, La Jolla Institute for Allergy and Immunology, 9420 Athena Circle, La Jolla, California 92037, USA. Phone: 858.752.6814; Email: toshi@liai.org.

JK's present address is: Department of Immunology, Graduate School of Pharmaceutical Sciences, Hokkaido University, Sapporo, Japan.

NIN's present address is: Department of Pediatrics, Shimoshizu National Hospital, Yotsukaido, Chiba, Japan.
1. Kagan RS. Food allergy: an overview. Environ Health Perspect. 2003;111(2):223-225.

2. Boyce JA, et al. Guidelines for the diagnosis and management of food allergy in the United States: summary of the NIAID-sponsored expert panel report. Nutr Res. 2011;31(1):61-75.

3. Jones SM, Burks AW, Dupont C. State of the art on food allergen immunotherapy: oral, sublingual, and epicutaneous. JAllergy Clin Immunol. 2014;133(2):318-323.

4. Wood RA. Food allergen immunotherapy: Current status and prospects for the future. JAllergy Clin Immunol. 2016;137(4):973-982.

5. Akdis CA, Akdis M. Mechanisms of allergenspecific immunotherapy and immune tolerance to allergens. World Allergy Organ J. 2015;8(1):17.

6. Yu W, Freeland DMH, Nadeau KC. Food allergy: immune mechanisms, diagnosis and immunotherapy. Nat Rev Immunol. 2016;16(12):751-765.

7. Helm RM. Food allergy animal models: an overview. Ann N Y Acad Sci. 2002;964:139-150.

8. Van Gramberg JL, de Veer MJ, O'Hehir RE, Meeusen EN, Bischof RJ. Use of animal models to investigate major allergens associated with food allergy. J Allergy (Cairo). 2013;2013:635695.

9. Brandt EB, et al. Mast cells are required for experimental oral allergen-induced diarrhea. J Clin Invest. 2003;112(11):1666-1677.

10. Forbes EE, et al. IL-9- and mast cell-mediated intestinal permeability predisposes to oral antigen hypersensitivity. J Exp Med. 2008;205(4):897-913.

11. Finkelman FD. Anaphylaxis: lessons from mouse models. JAllergy Clin Immunol. 2007;120(3):506-5015; quiz 516.

12. Kawakami T, Blank U. From IgE to Omalizumab. J Immunol. 2016;197(11):4187-4192.

13. Schneider LC, Rachid R, LeBovidge J, Blood E, Mittal M, Umetsu DT. A pilot study of omalizumab to facilitate rapid oral desensitization in high-risk peanut-allergic patients. J Allergy Clin Immunol. 2013;132(6):1368-1374.

14. Bommer UA. Cellular function and regulation of the translationally controlled tumor protein TCTP. The Open Allergy Journal. 2012;5:19-32.

15. Kawakami T, Kashiwakura J, Kawakami Y. Histamine-releasing factor and immunoglobulins in asthma and allergy. Allergy Asthma Immunol Res. 2014;6(1):6-12.
16. MacDonald SM, Rafnar T, Langdon J, Lichtenstein LM. Molecular identification of an IgEdependent histamine-releasing factor. Science. 1995;269(5224):688-690.

17. Warner JA, Pienkowski MM, Plaut M, Norman PS, Lichtenstein LM. Identification of histamine releasing factor(s) in the late phase of cutaneous IgE-mediated reactions. JImmunol. 1986;136(7):2583-2587.

18. MacDonald SM, et al. Studies of IgE-dependent histamine releasing factors: heterogeneity of $\operatorname{IgE}$ J Immunol. 1987;139(2):506-512.

19. Kashiwakura JC, et al. Histamine-releasing factor has a proinflammatory role in mouse models of asthma and allergy. J Clin Invest. 2012;122(1):218-228.

20. Chen CY, et al. Induction of interleukin-9producing mucosal mast cells promotes susceptibility to IgE-mediated experimental food allergy. Immunity. 2015;43(4):788-802.

21. Noti M, et al. Exposure to food allergens through inflamed skin promotes intestinal food allergy through the thymic stromal lymphopoietinbasophil axis. JAllergy Clin Immunol. 2014;133(5):1390-1399, 1399.e1.

22. Daëron M, Malbec O, Latour S, Arock M, Fridman WH. Regulation of high-affinity IgE receptor-mediated mast cell activation by murine low-affinity IgG receptors. J Clin Invest. 1995;95(2):577-585.

23. Uermösi C, et al. Mechanisms of allergen-specific desensitization. JAllergy Clin Immunol. 2010;126(2):375-383.

24. Tsai SH, et al. The ectoenzyme E-NPP3 negatively regulates ATP-dependent chronic allergic responses by basophils and mast cells. Immunity 2015;42(2):279-293.

25. Bowler RP, Crapo JD. Oxidative stress in allergic respiratory diseases. J Allergy Clin Immunol. 2002;110(3):349-356.

26. Kelly FJ, Sandström T. Air pollution, oxidative stress, and allergic response. Lancet. 2004;363(9403):95-96.

27. Verjan Garcia N, et al. SIRPa/CD172a regulates eosinophil homeostasis. JImmunol. 2011;187(5):2268-2277.

28. Itoh-Nagato $\mathrm{N}$, et al. Desensitization to a whole egg by rush oral immunotherapy improves the quality of life of guardians: A multicenter [pub- lished online ahead of print August 1, 2017]. Allergol Int. https://doi.org/10.1016/j.alit.2017.07.007.

29. Sato S, Yanagida N, Ohtani K, Koike Y, Ebisawa $\mathrm{M}$. A review of biomarkers for predicting clinical reactivity to foods with a focus on specific immunoglobulin E antibodies. Curr Opin Allergy Clin Immunol. 2015;15(3):250-258.

30. Yamashita H, Takahashi K, Tanaka H, Nagai H, Inagaki N. Overcoming food allergy through acquired tolerance conferred by transfer of Tregs in a murine model. Allergy. 2012;67(2):201-209.

31. Chen SH, et al. A knockout mouse approach reveals that TCTP functions as an essential factor for cell proliferation and survival in a tissueor cell type-specific manner. Mol Biol Cell. 2007;18(7):2525-2532.

32. Susini L, et al. TCTP protects from apoptotic cell death by antagonizing bax function. Cell Death Differ. 2008;15(8):1211-1220.

33. Sampson HA, Broadbent KR, Bernhisel-Broadbent J. Spontaneous release of histamine from basophils and histamine-releasing factor in patients with atopic dermatitis and food hypersensitivity. N Engl JMed.1989;321(4):228-232.

34. Bryce PJ, Miller ML, Miyajima I, Tsai M, Galli SJ, Oettgen HC. Immune sensitization in the skin is enhanced by antigen-independent effects of IgE. Immunity. 2004;20(4):381-392.

35. Burton OT, et al. Immunoglobulin E signal inhibition during allergen ingestion leads to reversal of established food allergy and induction of regulatory T cells. Immunity. 2014;41(1):141-151.

36. Gould HJ, et al. The biology of IGE and the basis of allergic disease. Annu Rev Immunol. 2003;21:579-628.

37. Cayrol C, Girard JP. IL-33: an alarmin cytokine with crucial roles in innate immunity, inflammation and allergy. Curr Opin Immunol. 2014;31:31-37.

38. Divekar R, Kita H. Recent advances in epitheliumderived cytokines (IL-33, IL-25, and thymic stromal lymphopoietin) and allergic inflammation. Curr Opin Allergy Clin Immunol. 2015;15(1):98-103.

39. Licona-Limón P, Kim LK, Palm NW, Flavell RA. TH2, allergy and group 2 innate lymphoid cells. Nat Immunol. 2013;14(6):536-542.

40. Martinez-Gonzalez I, Steer CA, Takei F. Lung ILC2s link innate and adaptive responses in allergic inflammation. Trends Immunol. 2015;36(3):189-195. 
41. Muto T, et al. The role of basophils and proallergic cytokines, TSLP and IL-33, in cutaneously sensitized food allergy. Int Immunol. 2014;26(10):539-549.

42. Han H, Thelen TD, Comeau MR, Ziegler SF. Thymic stromal lymphopoietin-medi- ated epicutaneous inflammation promotes acute diarrhea and anaphylaxis. JClin Invest. 2014;124(12):5442-5452.

43. Lee JB, et al. IL-25 and CD4(+) TH2 cells enhance type 2 innate lymphoid cell-derived IL-13 production, which promotes IgE-mediated experimental food allergy. J Allergy Clin Immunol. 2016;137(4):1216-1225.e5.

44. Doyle AD, et al. Homologous recombination into the eosinophil peroxidase locus generates a strain of mice expressing Cre recombinase exclusively in eosinophils. J Leukoc Biol. 2013;94(1):17-24. 